\title{
Probabilistic Collision Avoidance for Long-term Space Encounters via Risk Selection
}

\author{
Romain Serra, Denis Arzelier, Mioara Joldes and Aude Rondepierre
}

\begin{abstract}
This paper deals with collision avoidance between two space objects involved in a long-term encounter, assuming Keplerian linearized dynamics. The primary object is an active spacecraft - able to perform propulsive maneuvers - originally set on a reference orbit. The secondary object - typically an orbital debris is passive and represents a threat to the primary. The collision avoidance problem addressed in this paper aims at computing a fuel-optimal, finite sequence of impulsive maneuvers performed by the active spacecraft such that instantaneous collision probability remains below a given threshold over the encounter and that the primary object goes back to its reference trajectory at the end of the mission. Two successive relaxations are used to turn the original hard chance-constrained problem into a deterministic version that can be solved using mixed-integer linear programming solvers. An additional contribution is to propose a new algorithm to compute probabilities for 3-D Gaussian random variables to lie in Euclidean balls, enabling us to numerically validate the computed maneuvers by efficiently evaluating the resulting instantaneous collision probabilities.
\end{abstract}

Romain Serra

CNRS, LAAS, 7 avenue du Colonel Roche, F-31400 Toulouse,

Université de Toulouse, INSA, LAAS, 7 avenue du Colonel Roche, F-31400 Toulouse,

e-mail: serra@laas.fr

Denis Arzelier

CNRS, LAAS, 7 avenue du Colonel Roche, F-31400 Toulouse,

Université de Toulouse, LAAS, 7 avenue du Colonel Roche, F-31400 Toulouse,

e-mail: arzelier@laas.fr

Mioara Joldes

CNRS, LAAS, 7 avenue du Colonel Roche, F-31400 Toulouse,

Université de Toulouse, LAAS, 7 avenue du Colonel Roche, F-31400 Toulouse,

e-mail: joldes@laas.fr

Aude Rondepierre

Université de Toulouse, Institut de Mathématiques de Toulouse, INSA, 135 avenue de Rangueil,

F-31400 Toulouse, e-mail: aude.rondepierre@insa-toulouse.fr 


\section{Introduction}

During the last decades, the number of space debris around the Earth has been continuously and rapidly increasing. It now represents the majority of orbital objects and is a real threat for operational spacecraft. To prevent potential collisions on an active satellite, one solution consists in performing one or several evasive maneuvers when the predicted risk is too high according to mission requirements. These requirements also impose to end within given bounds relatively to the reference orbit by the time the threat is gone. This may call for one or several recovery maneuvers. In addition, the design of the overall thrust strategy is driven by the need to minimize fuel consumption in order to preserve the expected lifetime of the satellite.

This study deals with the collision avoidance problem between two orbiting objects involved in a long-term encounter, assuming a Keplerian linearized relative motion. One of the object, called the primary object and denoted by $p$, is an operational satellite initially set on a reference orbit. It is active in the sense that it is able to perform propulsive maneuvers to change its own trajectory. The second object, denoted by $s$, is passive: it is typically an uncontrolled space debris. The long-term encounter framework means that the time spent by the two space objects in the encounter region is large enough: it can extend up to a few orbital periods and corresponds to a relative velocity well below the threshold of the km.s ${ }^{-1}$.

Due to the lack of precision in measurements, the position and the velocity of each object are known with a certain amount of uncertainty, so they can be modeled as random variables. The collision risk is then quantified in terms of probability. The collision avoidance problem is formulated as a joint chance constrained optimization problem: it consists in computing a fuel optimal, finite sequence of impulsive maneuvers performed by the active spacecraft such that the probability of collision between the two objects does not exceed a user-defined threshold and that the primary object goes back to its reference orbit in due time. This paper aims at finding a practically solvable formulation for this optimization problem.

Since the original joint chance-constrained problem is very difficult to solve, simplifying assumptions are usually made to reduce the size of the optimization space or even to avoid the probabilistic formulation. In [15] the execution times and the thrust directions are heuristically fixed a priori so that the collision avoidance maneuvers are only optimized in magnitude. In [11], the avoidance strategy is reduced to one single maneuver. The maneuver direction is decoupled from its magnitude which allows to reduce the decision variables from three to one. In the context of satellites in formation flight, [17] proposes a simple strategy consisting in computing one velocity correction to achieve a fixed miss distance between the two objects. Note also the work in [10], where the collision avoidance problem is tackled as a robust optimization problem and in which safe guidance algorithms are designed for the PRISMA mission in the form of linear programs. Most of the time, the proposed strategies do not take any recovery maneuver to the reference orbit into account.

In this paper, the main idea is to relax the initial joint chance constrained optimization problem into a deterministic disjunctive linear program that can be solved for instance with a mixed-integer linear programming solver. To this end, two mech- 
anisms are used: (i) the collision set, usually modeled as a spherical geometrical object [5], is outer approximated here by a convex polyhedron; (ii) the so-called risk selection method, introduced first in [3] for probabilistic path planning. The degree of conservatism of these relaxations will be estimated in the chosen test case presented in the last section of this paper. The first contribution of this article is to propose an effective solution to the collision avoidance problem that takes recovery maneuvers to the reference orbit into account. The second contribution is to propose a new algorithm to efficiently compute the probability for any 3-D Gaussian random variable to lie in a Euclidean ball. This is an extension of the 2-D method described in [16]. This efficient evaluation of the instantaneous probability of collision is used here to analyze $a$ posteriori the computed maneuvers and to validate on a finer grid the mitigation of the collision risk.

Notations: Capital letters are dedicated to random variables and small characters to instances of these variables. Let $Y$ be a random vector: $y$ is an occurrence of $Y, \mu_{Y}$ the mean vector of $Y$ and $\Sigma_{Y}$ its variance-covariance matrix. Finally, the $d$ dimensional normal (or Gaussian) distribution of a $d$-dimensional random vector $Y$ is denoted by: $Y \sim \mathscr{N}_{d}\left(\mu_{Y}, \Sigma_{Y}\right) . \mathscr{P}(\{\cdot\})$ is the probability of the event $\{\cdot\}$.

\section{Probabilistic model for collision avoidance}

Two spherical space objects involved in a long-term encounter over a time horizon $\left[t_{0}, t_{f}\right]$ are considered. This section addresses the mathematical formulation of the problem of a long-term encounter between these two orbiting objects. Let us first introduce the Local Vertical Local Horizontal (LVLH) orbital frame $\left(O_{\tilde{p}}, \mathbf{u}_{\mathbf{x}}, \mathbf{u}_{\mathbf{y}}, \mathbf{u}_{\mathbf{z}}\right)$ attached to the nominal orbit of the primary object (see Figure 1):

- Origin $O_{\tilde{p}}$ : nominal position of the primary center of mass;

- $Z$ axis (R-bar): radial direction (Nadir-Target), oriented towards the center of the Earth;

- $Y$ axis (H-bar): perpendicular to the nominal primary orbital plane, pointing opposite the angular momentum;

- $X$ axis (V-bar): chosen such that $\mathbf{u}_{\mathbf{x}}=\mathbf{u}_{\mathbf{y}} \times \mathbf{u}_{\mathbf{z}}$.

This frame is used for the linearized equations of motion in what follows.

\subsection{Model of the linearized dynamics}

The long-term encounter assumption means that the time spent by the two objects in the encounter region is significant. One can then reasonably assume that during the encounter the relative distances of the two objects to the reference position are small compared to their distance to the Earth. Under this assumption, the respective equations of motion for each object may be linearized around the reference orbit. 


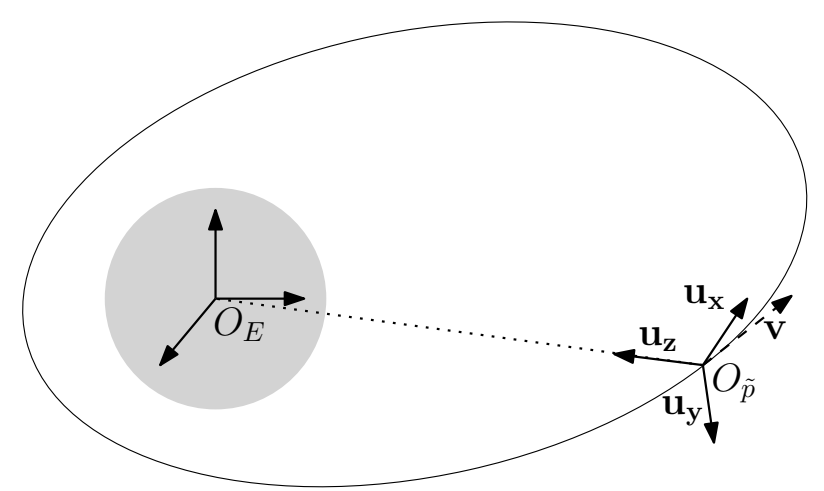

Fig. 1 Local Vertical Local Horizontal (LVLH) frame

Assuming unperturbed Keplerian motions, the linearized equations are described by the standard Tschauner-Hempel equations [18] and its associated YamanakaAnkersen's state transition matrix [20].

Let $\mathbf{x}_{p}=\left[\begin{array}{r}\mathbf{r}_{p} \\ \mathbf{v}_{p}\end{array}\right]^{T}$ and $\mathbf{x}_{s}=\left[\mathbf{r}_{s} \mathbf{v}_{s}\right]^{T}$ respectively denote the position and velocity vectors of the primary and the secondary objects. Unlike the secondary object, the primary object can use its thrusters to modify its trajectory. These propulsive maneuvers are assumed to be impulsive. The impulsive approximation for the thrust means that instantaneous velocity increments are applied to the primary object instead of finite-thrust powered phases of finite duration. Let $\hat{T}=\left\{\hat{t}_{1}, \hat{t}_{2} \ldots, \hat{t}_{N_{1}}\right\}$ be a sorted set of dates in $\left[t_{0}, t_{f}\right]$ when a maneuver can be performed. For each $i \in\left\{1, \ldots, N_{1}\right\}$, the impulsive assumption could be expressed as:

$$
\mathbf{x}_{p}\left(\hat{t}_{i}^{+}\right)=\mathbf{x}_{p}\left(\hat{t}_{i}^{-}\right)+B \Delta V_{i}
$$

where $B=\left[\begin{array}{c}0_{3} \\ I_{3}\end{array}\right]$ and $\Delta V_{i}$ is the velocity increment applied at $\hat{t}_{i}$.

Under the previous assumptions and considering that the primary object $p$ is actuated while $s$ is not, propagation of the state vectors $\mathbf{x}_{p}$ and $\mathbf{x}_{s}$ follow equations (2) and (3):

$$
\begin{aligned}
& \mathbf{x}_{p}(t)=\Phi\left(t, t_{0}\right) \mathbf{x}_{p}^{0}+\sum_{\substack{i \in\left\{1, \ldots, N_{1}\right\} \\
\hat{t}_{i} \leq t}} \Phi\left(t, \hat{t}_{i}\right) B \Delta V_{i} \\
& \mathbf{x}_{s}(t)=\Phi\left(t, t_{0}\right) \mathbf{x}_{s}^{0},
\end{aligned}
$$

where $\mathbf{x}_{p}^{0}=\mathbf{x}_{p}\left(t_{0}\right), \mathbf{x}_{s}^{0}=\mathbf{x}_{s}\left(t_{0}\right)$ and $\Phi(\cdot, \cdot)$ is the Yamanaka-Ankersen transition matrix. The set $\hat{T}$ of dates is assumed fixed a priori. Its choice can follow from considerations on heuristics or previous runs of the algorithm. By default, it can simply be a uniform discretization of $\left[t_{0}, t_{f}\right]$.

It is also assumed that the primary object $p$ is actuated by identical engines rigidly mounted to the body axes of the spacecraft; consequently the fuel consumption will 
be defined in the sequel as:

$$
J=\sum_{i=1}^{N_{1}}\left\|\Delta V_{i}\right\|_{1}
$$

Note that a second time grid $T=\left\{t_{0}<t_{1}<t_{2}<\ldots<t_{N_{2}}=t_{f}\right\}$, uniform and thinner than $\hat{T}$ (i.e. $N_{2}>N_{1}$ ) is introduced to evaluate the instantaneous probability of collision in the problem of collision avoidance.

\subsection{Uncertainty on positions and velocities}

Due to the current limitations of orbit acquisition systems, information about the objects trajectories and velocities is known with a certain amount of uncertainty. Therefore, positions and velocities of the two objects at initial time $t_{0}$ are not known exactly and can be seen as jointly distributed random variables, respectively denoted by $\mathbf{X}_{p}^{0}$ and $\mathbf{X}_{s}^{0}$, following independent Gaussian distribution laws:

$$
\mathbf{X}_{p}^{0} \sim \mathscr{N}_{6}\left(\mu_{\mathbf{X}_{p}^{0}}, \Sigma_{\mathbf{X}_{p}^{0}}\right), \quad \mathbf{X}_{s}^{0} \sim \mathscr{N}_{6}\left(\mu_{\mathbf{X}_{s}^{0}}, \Sigma_{\mathbf{X}_{s}^{0}}\right) .
$$

Under these assumptions, the relative position and velocity vector at time $t_{0}$ is also a Gaussian random vector, denoted by $\mathbf{X}^{0}=\mathbf{X}_{p}^{0}-\mathbf{X}_{s}^{0}$ :

$$
\mathbf{X}^{0} \sim \mathscr{N}_{6}\left(\mu_{\mathbf{X}_{p}^{0}}-\mu_{\mathbf{X}_{s}^{0}}, \Sigma_{\mathbf{X}_{p}^{0}}+\Sigma_{\mathbf{X}_{s}^{0}}\right) .
$$

Let us now focus on the propagation of uncertainties over the time horizon $\left[t_{0}, t_{f}\right]$. Let $\mathbf{X}_{p}^{k}$ and $\mathbf{X}_{s}^{k}$ be the random state vectors at time $t_{k}$. Due to the linear setting, the Gaussian nature of uncertainty is preserved over the time horizon $\left[t_{0}, t_{f}\right]$. In other words, at any time $t_{k}, k \in\left\{1, \ldots, N_{2}\right\}$, one has:

$$
\mathbf{X}_{p}^{k} \sim \mathscr{N}_{6}\left(\mu_{\mathbf{X}_{p}^{k}}, \Sigma_{\mathbf{X}_{p}^{k}}\right), \quad \mathbf{X}_{s}^{k} \sim \mathscr{N}_{6}\left(\mu_{\mathbf{X}_{s}^{k}}, \Sigma_{\mathbf{X}_{s}^{k}}\right) .
$$

Using the propagation equations (2) and (3), the mean and the covariance matrices can be easily expressed as:

$$
\begin{array}{ll}
\mu_{\mathbf{X}_{p}^{k}}=\Phi\left(t_{k}, t_{0}\right) \mu_{\mathbf{X}_{p}^{0}}+\sum_{\substack{i \in\left\{1, \ldots, N_{1}\right\} \\
\hat{t}_{i} \leq t_{k}}} \Phi\left(t_{k}, \hat{t}_{i}\right) B \Delta V_{i}, & \mu_{\mathbf{X}_{s}^{k}}=\Phi\left(t_{k}, t_{0}\right) \mu_{\mathbf{X}_{s}^{0}}, \\
\Sigma_{\mathbf{X}_{p}^{k}}=\Phi\left(t_{k}, t_{0}\right) \Sigma_{\mathbf{X}_{p}^{0}} \Phi\left(t_{k}, t_{0}\right)^{T}, & \Sigma_{\mathbf{X}_{s}^{k}}=\Phi\left(t_{k}, t_{0}\right) \Sigma_{\mathbf{X}_{s}^{0}} \Phi\left(t_{k}, t_{0}\right)^{T} .
\end{array}
$$

The relative position and velocity random vector $\mathbf{X}^{k}=\mathbf{X}_{p}^{k}-\mathbf{X}_{s}^{k}$ at time $t_{k}$ is then defined by:

$$
\mathbf{X}^{k} \sim \mathscr{N}_{6}\left(\mu_{\mathbf{X}^{k}}, \Sigma_{\mathbf{X}^{k}}\right)
$$

where: 


$$
\begin{aligned}
& \mu_{\mathbf{X}^{k}}=\Phi\left(t_{k}, t_{0}\right)\left(\mu_{\mathbf{X}_{p}^{0}}-\mu_{\mathbf{X}_{s}^{0}}\right)+\sum_{\substack{i \in\left\{1, \ldots, N_{1}\right\} \\
\hat{t}_{i} \leq t_{k}}} \Phi\left(t_{k}, \hat{t}_{i}\right) B \Delta V_{i}, \\
& \Sigma_{\mathbf{X}^{k}}=\Phi\left(t_{k}, t_{0}\right)\left(\Sigma_{\mathbf{X}_{p}^{0}}+\Sigma_{\mathbf{X}_{s}^{0}}\right) \Phi\left(t_{k}, t_{0}\right)^{T} .
\end{aligned}
$$

Note that the respective probability distributions for position and velocity at any time - as marginals of Gaussian laws - are Gaussian too. Their respective covariance matrices can be simply obtained by taking the adequate $3 \times 3$ submatrices of the $6 \times 6$ matrices describing the complete states.

\subsection{Formulation of the collision avoidance problem}

The collision avoidance problem considered in this paper, is to compute a fueloptimal, finite sequence of impulsive maneuvers performed by the active spacecraft $p$ such that the instantaneous probability of collision between the two objects does not exceed some user-defined tolerance threshold $\delta$. In addition, the primary object has to be back on its reference orbit at final time $t_{f}$.

This very last constraint is a key feature of the proposed approach. To ensure that the primary object goes back to its reference orbit at final time $t_{f}$, the following boundary condition is imposed: $\mu_{\mathbf{X}_{p}^{N}}=0$, i.e.:

$$
\Phi\left(t_{f}, t_{0}\right) \mu_{\mathbf{X}_{p}^{0}}+\sum_{i=1}^{N_{1}} \Phi\left(t_{f}, \hat{t}_{i}\right) B \Delta V_{i}=0,
$$

which is a linear constraint in the decision variables $\Delta V_{i}, i=1, \ldots, N_{1}$.

In the literature, the orbiting objects are often assumed to have a spherical shape [5]. This assumption enables to ignore any constraint on the attitude and to give a rough model of objects whose geometry is not very precisely known. Under this assumption, the collision set in the relative position space, referred to as the combined object, is defined as:

$$
\left\{\mathbf{r} \in \mathbb{R}^{3}:\|\mathbf{r}\|_{2} \leq R\right\},
$$

where $R$ is the sum of the radii of the two objects. The collision avoidance problem can then be formulated as:

$$
\min _{\Delta V_{i}} \sum_{i=1}^{N_{1}}\left\|\Delta V_{i}\right\|_{1} \text { s.t. } \mid \begin{aligned}
& \mathscr{P}\left(\left\{\left\|\mathbf{R}^{k}\right\|_{2} \leq R\right\}\right) \leq \delta, k=1, \ldots, N_{2}, \\
& \Phi\left(t_{f}, t_{0}\right) \mu_{\mathbf{X}_{p}^{0}}+\sum_{i=1}^{N_{1}} \Phi\left(t_{f}, \hat{t}_{i}\right) B \Delta V_{i}=0 .
\end{aligned}
$$

where: $\mathbf{R}^{k}=\left(\begin{array}{ll}I_{3} & 0\end{array}\right) \mathbf{X}^{k}$ denotes the relative position random vector.

Remark 1. Rigorously speaking, instead of the instantaneous collision probability, one should consider the probability of collision over the whole time horizon as a 
constraint. However, despite the simplifying assumptions used here, this quantity is very difficult to manipulate: relative dynamics are still fairly complex and the set of initial conditions leading to a collision occurring before $t_{f}$, is difficult to derive.

For any $k \in\left\{1, \ldots, N_{2}\right\}$, the instantaneous collision probability $\mathscr{P}\left(\left\{\left\|\mathbf{R}^{k}\right\|_{2} \leq R\right\}\right)$ at time $t_{k}$ is the integral of the Gaussian probability density function of $\mathbf{R}^{k}$ over this ball. This integral can be computed in a fast and efficient way as shown in Section 2 , but is hard to handle as a constraint in problem (8) due to its lack of particular properties with respect to the decision variables.

This section is dedicated to the reformulation of (8) into a more tractable optimization problem. This is done in two steps. Firstly, a conservative polyhedral approximation of the spherical combined object is proposed in Paragraph 1.3.1: the collision set is described by a finite number of equations that are linear in the control vector, and resulting in linear chance constraints that are jointly defined. Then, the so-called risk selection method is used in Paragraph 1.3.2 to replace them by individual probabilistic constraints. The induced individual chance constrained problem is then equivalently reformulated as a deterministic optimization problem.

\subsubsection{Polyhedral approximation of the collision set}

In this section, the collision set $\left\{\mathbf{r} \in \mathbb{R}^{3}:\|\mathbf{r}\|_{2} \leq R\right\}$ is conservatively replaced by a (convex) polyhedron containing it. Recall that a convex polyhedron in $\mathbb{R}^{3}$ is defined by a finite number of affine inequalities, or equivalently as the intersection of half-spaces. The simplest example of a convex polyhedron containing the spherical combined object, is a cube whose faces are tangent to it (see Figure 2).

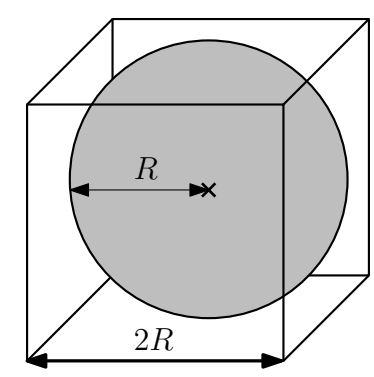

Fig. 2 Combined spherical object included in a cube

More generally, assume that the collision set is included in a given polyhedron:

$$
\left\{\mathbf{r} \in \mathbb{R}^{3}:\|\mathbf{r}\|_{2} \leq R\right\} \subset\left\{\mathbf{r} \in \mathbb{R}^{3}: \bigcap_{j=1}^{m}\left(\mathbf{a}_{j}^{T} \mathbf{r} \leq b_{j}\right)\right\},
$$

where $\mathbf{a}_{j} \in \mathbb{R}^{3}, b_{j} \in \mathbb{R}, j=1, \ldots, m$. Then: 


$$
\mathscr{P}\left(\left\{\left\|\mathbf{R}^{k}\right\| \leq R\right\}\right) \leq \mathscr{P}\left(\bigcap_{j=1}^{m}\left\{a_{j}^{T} \mathbf{R}^{k} \leq b_{j}\right\}\right) .
$$

Using the polyhedral approximation (9), the collision avoidance problem is then replaced by:

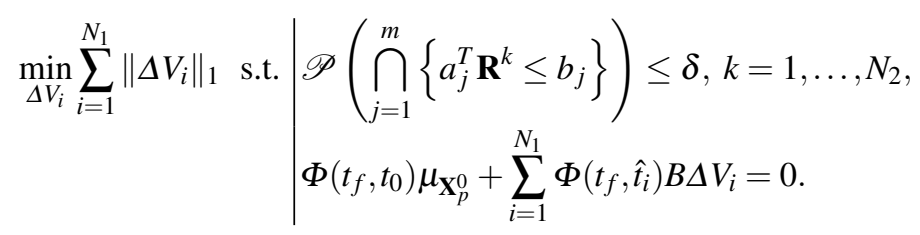

The problem (10) is a joint chance constrained optimization problem which is a relaxation of (8). This approximation is conservative in the sense that any solution of problem (10) is also a solution of (8).

Note that the degree of conservatism is closely related to the tightness of the geometrical approximation between the collision set (7) and its polyhedral approximation (9). There is a clear trade-off between increasing the complexity of the chosen polyhedron by increasing the number of inequalities defining the polyhedron and the computational complexity of the considered optimization problem.

\subsubsection{Towards a deterministic formulation}

In this section, the so-called risk selection method is used to replace the joint chance constraints in (10) by individual chance constraints. This technique was first used in [3] for probabilistic path planning although the name only appeared later (see e.g. [4]). Let us focus on the instantaneous probability of collision at a given time step $t_{k}$ defined by:

$$
\mathscr{P}_{c}^{k}=\mathscr{P}\left(\bigcap_{j=1}^{m}\left\{\mathbf{a}_{j}^{T} \mathbf{R}^{k} \leq b_{j}\right\}\right) .
$$

One has:

$$
\mathscr{P}_{c}^{k} \leq \mathscr{P}\left(\left\{\mathbf{a}_{j}^{T} \mathbf{R}^{k} \leq b_{j}\right\}\right) \quad \forall j=1, \ldots, m,
$$

which means that if there exists $j \in\{1, \ldots, m\}$ such that $\mathscr{P}\left(\left\{\mathbf{a}_{j}^{T} \mathbf{R}^{k} \leq b_{j}\right\}\right) \leq \delta$ then $\mathscr{P}_{c}^{k} \leq \delta$. In other words:

$$
\bigvee_{j=1}^{m}\left(\mathscr{P}\left(\left\{\mathbf{a}_{j}^{T} \mathbf{R}^{k} \leq b_{j}\right\}\right) \leq \delta\right) \Longrightarrow \mathscr{P}_{c}^{k} \leq \delta,
$$

where $\bigvee$ is the disjunction symbol. The original joint chance constraint $\mathscr{P}_{c}^{k} \leq \delta$ can thus be conservatively replaced by the following disjunctive set of individual constraints: 


$$
\bigvee_{j=1}^{m}\left(\mathscr{P}\left(\left\{\mathbf{a}_{j}^{T} \mathbf{R}^{k} \leq b_{j}\right\}\right) \leq \delta\right) .
$$

This approach is called risk selection because instead of enforcing the chance for the relative position vector to lie outside the collision set, one enforces it to be in one of the half-spaces outside the convex polyhedron.

After these two successive relaxations (the polyhedral approximation and the risk selection method), the collision avoidance problem (10) is cast as the following individual chance constrained problem:

$$
\min _{\Delta V_{i}} \sum_{i=1}^{N_{1}}\left\|\Delta V_{i}\right\|_{1} \text { s.t. } \mid \begin{aligned}
& \bigvee_{j=1}^{m}\left(\mathscr{P}\left(\left\{\mathbf{a}_{j}^{T} \mathbf{R}^{k} \leq b_{j}\right\}\right) \leq \delta\right), k=1, \ldots, N_{2}, \\
& \Phi\left(t_{f}, t_{0}\right) \mu_{\mathbf{X}_{p}^{0}}+\sum_{i=1}^{N_{1}} \Phi\left(t_{f}, \hat{t}_{i}\right) B \Delta V_{i}=0 .
\end{aligned}
$$

The new constraint (14) is an individual chance constraint, but remains probabilistic. It can be equivalently reformulated into a deterministic form following [3].

Let $Y$ be a one-dimensional random variable following a Gaussian distribution law: $Y \sim \mathscr{N}_{1}\left(\mu_{Y}, \sigma_{Y}^{2}\right)$. Any chance constraint on $Y$ with a fixed variance and a variable mean, can be translated into a deterministic constraint on the mean $\mu_{Y}$ (see [3, Section III.C]):

$$
\mathscr{P}(\{Y<0\}) \leq \delta \Longleftrightarrow \mu_{Y} \geq \sqrt{2} \sigma_{Y} \operatorname{erf}^{-1}(1-2 \delta),
$$

where erf is the error function defined by: $\operatorname{erf}(z)=\frac{2}{\sqrt{\pi}} \int_{0}^{z} e^{-t^{2}} d t$. By applying (16) to $Y=\mathbf{a}_{j}^{T} \mathbf{R}^{k}-b_{j} \sim \mathscr{N}_{1}\left(\mathbf{a}_{j}^{T} \mu_{\mathbf{R}^{k}}-b_{j}, \mathbf{a}_{j}^{T} \Sigma_{\mathbf{R}^{\mathbf{k}}} \mathbf{a}_{j}\right)$, each individual probabilistic constraint can be equivalently replaced by an affine inequality on the mean:

$$
\mathscr{P}\left(\left\{\mathbf{a}_{j}^{T} \mathbf{R}^{k} \leq b_{j}\right\}\right) \leq \delta \Longleftrightarrow \mathbf{a}_{j}^{T} \mu_{\mathbf{R}^{k}} \geq b_{j}+c_{j k},
$$

where: $c_{j k}=\sqrt{2 \mathbf{a}_{j}^{T} \Sigma_{\mathbf{R}^{k}} \mathbf{a}_{j}} \operatorname{erf}^{-1}(1-2 \delta)$. In conclusion, the collision avoidance problem (15) can be equivalently reformulated as:

$$
\min _{\Delta V_{i}} \sum_{i=1}^{N_{1}}\left\|\Delta V_{i}\right\|_{1} \text { s.t. } \mid \begin{aligned}
& \bigvee_{j=1}^{m}\left(\mathbf{a}_{j}^{T} \mu_{\mathbf{R}^{k}} \geq b_{j}+c_{j k}\right), \forall k=1, \ldots, N_{2}, \\
& \Phi\left(t_{f}, t_{0}\right) \mu_{\mathbf{X}_{p}^{0}}+\sum_{i=1}^{N_{1}} \Phi\left(t_{f}, \hat{t}_{i}\right) B \Delta V_{i}=0 .
\end{aligned}
$$




\subsection{Towards a Mixed-Integer Linear Programming Problem}

In the previous section, a tractable deterministic formulation (17) of the collision avoidance problem involving disjunctive constraints, has been derived. Let us now show that the latter is actually a mixed integer linear program which can be solved using dedicated algorithms.

First, in order to get rid of piecewise linear criterion, usual slack variables $\Delta V_{i}^{-}, \Delta V_{i}^{+}$belonging to the positive orthant of $\mathbb{R}^{3}$ are introduced [2]: for all $i \in\left\{1, \ldots, N_{1}\right\}$, the following substitutions are done:

$$
\Delta V_{i}=\Delta V_{i}^{+}-\Delta V_{i}^{-}
$$

Note that the original piecewise linear criterion $\sum_{i=1}^{N_{1}}\left\|\Delta V_{i}\right\|_{1}$ may be equivalently replaced by the linear criterion $\sum_{i=1}^{N_{1}}\left(\left\|\Delta V_{i}^{+}\right\|_{1}+\left\|\Delta V_{i}^{-}\right\|_{1}\right)=\sum_{i=1}^{N_{1}} \sum_{j=1}^{3}\left(\Delta V_{i}^{+j}+\Delta V_{i}^{-j}\right)$ since at the optimum $\Delta V_{i}^{+j}=0$ or $\Delta V_{i}^{-j}=0$ [2].

The disjunctive constraints are then tackled using the classic big M method [12]. For the clarity of the presentation, this method is recalled on a one-dimensional example. Let us consider the following two linear disjunctive conditions in the scalar variable $y$ :

$$
y \leq y_{\min } \text { or } y \geq y_{\max } .
$$

If the scalar $M$ is chosen to be sufficiently large, i.e. larger than any other quantity of the problem, the previous conditions are equivalent to the following ones:

$$
y \leq y_{\min }+q M \quad \text { and } \quad-y \leq-y_{\max }+M q^{\prime} \quad \text { and } \quad q+q^{\prime} \leq 1,
$$

where $q$ and $q^{\prime}$ are binary variables: $q, q^{\prime} \in\{0,1\}$. Any linear program involving such disjunctive constraints, may be recast into a Mixed-Integer Linear Program (MILP). In our case, it leads to an optimization problem with $2 \times 3 \times N_{1}=6 N_{1}$ continuous variables and $m \times N_{2}$ binary variables:

$$
\min _{\Delta V_{i}^{ \pm}, q_{k} \sum_{i=1}^{N_{1}}\left(\left\|\Delta V_{i}^{+}\right\|_{1}+\left\|\Delta V_{i}^{-}\right\|_{1}\right) \text { s.t. }} \begin{aligned}
& \bigwedge_{j=1}^{m}\left(\mathbf{a}_{j}^{T} \mu_{\mathbf{R}^{k}}+M q_{k}^{j} \geq b_{j}+c_{j k}\right), \forall k=1, \ldots, N_{2}, \\
& \Phi\left(t_{f}, t_{0}\right) \mu_{\mathbf{X}_{p}^{0}}+\sum_{i=1}^{N_{1}} \Phi\left(t_{f}, \hat{t}_{i}\right) B\left(\Delta V_{i}^{+}-\Delta V_{i}^{-}\right)=0, \\
& \left\|\mathbf{q}_{k}\right\|_{1} \leq m-1, k=1, \ldots, N_{2}, \\
& \Delta V_{i}^{ \pm} \geq 0, i=1, \ldots, N_{1}, \\
& \mathbf{q}_{k} \in\{0,1\}^{m}, k=1, \ldots, N_{2} .
\end{aligned}
$$

Note that in the proposed approach, there is no guarantee for the instantaneous collision probability to be below a given threshold between the points of the time grid $T$. To alleviate this limitation, a precise a posteriori analysis of the computed ma- 
neuvers is performed by computing the original instantaneous collision probability in (8) over a thinner grid than the one used in (19).

\section{Computing the instantaneous probability of collision}

In the modeling of the collision avoidance problem, the instantaneous probability of collision $\mathscr{P}\left(\|\mathbf{R}\|_{2} \leq R\right)$ has to be smaller than a given threshold $\delta$ at any instant $t_{k}$ of a given time discretization of $\left[t_{0}, t_{f}\right]$. To validate this approach, one needs now to compute the instantaneous probability of collision at each time $t \in\left[t_{0}, t_{f}\right]$ to numerically verify that it remains below the threshold $\delta$ over the whole time interval $\left[t_{0}, t_{f}\right]$ :

$$
\forall t \in\left[t_{0}, t_{f}\right], \mathscr{P}\left(\left\{\|\mathbf{R}(t)\|_{2} \leq R\right\}\right) \leq \delta .
$$

In that purpose, the conservative polyhedral model is not needed any more and the spherical combined object can be considered again. Under this assumption, the instantaneous probability of collision can be expressed as the integral of a 3-D Gaussian random variable - representing the current relative position of the two objects on a Euclidean ball. Usually, that kind of integral is evaluated using time-consuming Monte Carlo methods. Here, an analytic formula based on a convergent power series is introduced. It is a 3-D extension to the 2-D method presented in [16]. It is derived by use of Laplace techniques originally developed in [9], and properties of D-finite functions [21,13]. Computing truncated series should allow for a fast evaluation of the instantaneous probability of collision.

Assuming that $\mathbf{R} \sim \mathscr{N}_{3}(\mu, \Sigma)$, the instantaneous probability of collision can be expressed as:

$$
\mathscr{P}=\frac{1}{(2 \pi)^{3 / 2}|\Sigma|^{1 / 2}} \int_{\mathscr{B}(0, R)} \exp \left(-\frac{1}{2}(r-\mu)^{T} \Sigma^{-1}(r-\mu)\right) \mathrm{d} r
$$

where $\mu \in \mathbb{R}^{3}$ and $\Sigma$ is a $3 \times 3$ positive definite matrix. Without loss of generality, the covariance matrix $\Sigma$ is assumed to be diagonal (this can be always achieved by a classical change of variables). Thus, $\Sigma$ can be written as:

$$
\Sigma=\operatorname{diag}\left(\sigma_{1}^{2}, \sigma_{2}^{2}, \sigma_{3}^{2}\right),
$$

where $\left(\sigma_{1}, \sigma_{2}, \sigma_{3}\right) \in \mathbb{R}_{*}^{+3}$, and: $\sigma_{1} \geq \sigma_{2} \geq \sigma_{3}$. Under these assumptions, the probability $\mathscr{P}$ can be rewritten as:

$$
\mathscr{P}=\frac{1}{(2 \pi)^{3 / 2} \sigma_{1} \sigma_{2} \sigma_{3}} \int_{\mathscr{B}(0, R)} \exp \left(-\frac{1}{2} \sum_{i=1}^{3} \frac{\left(r_{i}-\mu_{i}\right)^{2}}{\sigma_{i}^{2}}\right) \mathrm{d} r .
$$

Both level sets of integrand and domain of integration can be visualized in Figure 3. 


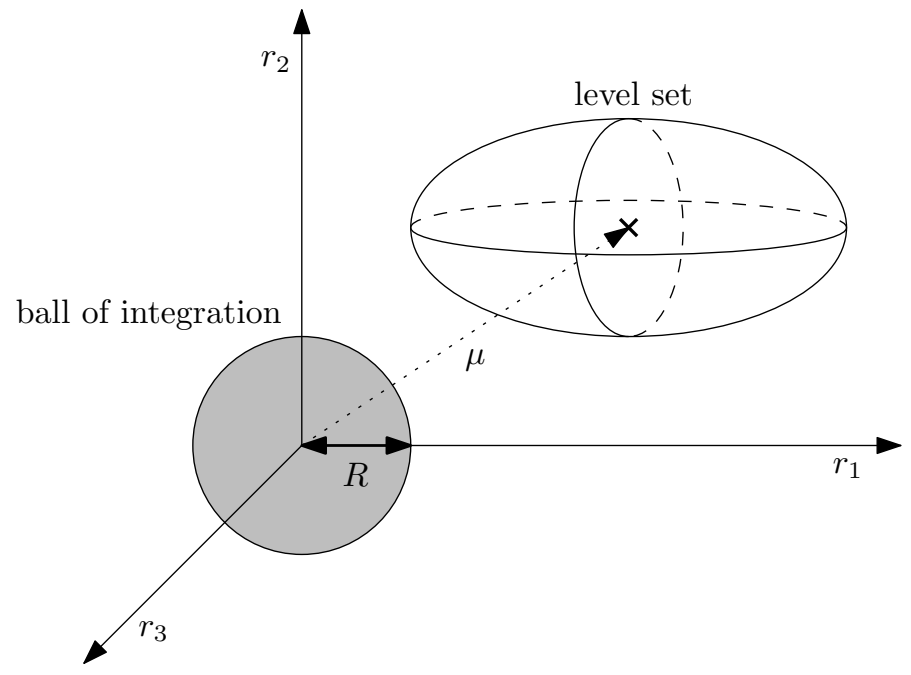

Fig. 3 Geometry of the integral problem

Proposition 1.

$$
\mathscr{P}=\exp \left(-\frac{R^{2}}{\sigma_{3}^{2}}\right) \sum_{k=0}^{+\infty} u_{k} R^{2 k},
$$

where the sequence $\left(u_{k}\right)_{k \geq 0}$ is positive and explicitly defined by a linear recurrence with polynomial coefficients.

\section{Sketch of the proof}

Let us introduce an intermediate function called $g$ and defined for all $z \in \mathbb{R}^{+}$as follows:

$$
g(z)=\frac{1}{(2 \pi)^{3 / 2} \sigma_{1} \sigma_{2} \sigma_{3}} \int_{\mathscr{B}(0, \sqrt{z})} \exp \left(-\frac{1}{2} \sum_{i=1}^{3} \frac{\left(r_{i}-\mu_{i}\right)^{2}}{\sigma_{i}^{2}}\right) \mathrm{d} r,
$$

so that the instantaneous probability of collision is given by: $\mathscr{P}=g\left(R^{2}\right)$. Using the set-indicator function $\mathbf{1}$, the function $g$ can also be rewritten as:

$$
g(z)=\frac{1}{(2 \pi)^{3 / 2} \sigma_{1} \sigma_{2} \sigma_{3}} \int_{\mathbb{R}^{3}} \mathbf{1}_{\mathscr{B}(0, \sqrt{z})}(r) \exp \left(-\frac{1}{2} \sum_{i=1}^{3} \frac{\left(r_{i}-\mu_{i}\right)^{2}}{\sigma_{i}^{2}}\right) \mathrm{d} r .
$$

The main idea of the proof is to compute the Laplace transform of the function $g$ in closed form, to expand it into a power series in the variable $\lambda^{-1}$ and to apply the 
inverse Laplace transform term by term. Finally, a preconditioning with an exponential term is performed in order to obtain a series with positive coefficients.

Let us first apply the Laplace transform to the function $g$ : for all $\lambda$ whose real part $\Re(\lambda)$ is non-negative, one has:

$$
\mathscr{L}_{g}(\lambda)=\int_{0}^{+\infty} \exp (-\lambda z) g(z) \mathrm{d} z
$$

The Laplace transform $\mathscr{L}_{g}$ can be computed in closed form. Starting from:

$$
\mathscr{L}_{g}(\lambda)=\frac{1}{(2 \pi)^{3 / 2} \sigma_{1} \sigma_{2} \sigma_{3}} \int_{0}^{+\infty} \int_{\mathbb{R}^{3}} \mathbf{1}_{\mathscr{B}(0, \sqrt{z})}(r) \exp \left(-\lambda z-\frac{1}{2} \sum_{i=1}^{3} \frac{\left(r_{i}-\mu_{i}\right)^{2}}{\sigma_{i}^{2}}\right) \mathrm{d} r \mathrm{~d} z
$$

the Fubini theorem enables us to interchange the two integration symbols:

$$
\mathscr{L}_{g}(\lambda)=\frac{1}{(2 \pi)^{3 / 2} \sigma_{1} \sigma_{2} \sigma_{3}} \int_{\mathbb{R}^{3}} \int_{0}^{+\infty} \mathbf{1}_{\mathscr{B}(0, \sqrt{z})}(r) \exp \left(-\lambda z-\frac{1}{2} \sum_{i=1}^{3} \frac{\left(r_{i}-\mu_{i}\right)^{2}}{\sigma_{i}^{2}}\right) \mathrm{d} z \mathrm{~d} r .
$$

Now, the integral in the variable $z$ can be computed analytically, so that:

$$
\mathscr{L}_{g}(\lambda)=\frac{1}{(2 \pi)^{3 / 2} \lambda \sigma_{1} \sigma_{2} \sigma_{3}} \int_{\mathbb{R}^{3}} \exp \left(\sum_{i=1}^{3}\left(-\left(\lambda+\frac{1}{2 \sigma_{i}^{2}}\right) r_{i}^{2}+\frac{\mu_{i} r_{i}}{\sigma_{i}^{2}}-\frac{\mu_{i}^{2}}{2 \sigma_{i}^{2}}\right)\right) \mathrm{d} r .
$$

The next step consists in doing a change of variables aimed at completing the square in the exponential. More precisely, state:

$$
r_{i}^{\prime}=r_{i}-\frac{\mu_{i}}{2 \sigma_{i}^{2}}\left(\lambda+\frac{1}{2 \sigma_{i}^{2}}\right)^{-1} \quad \forall i=1,2,3 .
$$

By computation, one finally gets:

$$
\mathscr{L}_{g}(\lambda)=\lambda^{-5 / 2} \exp \left(-\sum_{i=1}^{3} \mu_{i}^{2}\left(2 \sigma_{i}^{2}+\lambda^{-1}\right)^{-1}\right) \prod_{i=1}^{3}\left(2 \sigma_{i}^{2}+\lambda^{-1}\right)^{-1 / 2} .
$$

Now $\lambda^{5 / 2} \mathscr{L}_{g}(\lambda)$ can be seen as a function of the variable $\lambda^{-1}$, which can be expanded in a power series $\sum_{k=0}^{+\infty} w_{k}\left(\lambda^{-1}\right)^{k}$. This is valid for $\Re(\lambda)>\frac{1}{2 \sigma_{3}^{2}}$. The sequence $\left(w_{k}\right)_{k \geq 0}$ follows a linear recurrence with polynomial terms in the variable $k$. This recurrence can be obtained from the differential equation satisfied by $\lambda^{-1} \rightarrow \lambda^{5 / 2} \mathscr{L}_{g}(\lambda)$. Indeed this function is D-finite i.e. is solution of a linear differential equation with polynomial coefficients. The Maple package Gfun [14](version 3.65 ) was used to obtain the recurrence formula. One can now write: 


$$
\mathscr{L}_{g}(\lambda)=\lambda^{-5 / 2} \sum_{k=0}^{+\infty} w_{k} \lambda^{-k}=\sum_{k=0}^{+\infty} w_{k} \lambda^{-(k+5 / 2)}
$$

Let us now apply the inverse Laplace transform to the previous expression. On the left hand side, one gets the function $g$ back. On the right hand side, the inverse Laplace transform is done term by term via the technique described by Widder [19]. It leads to the introduction of the gamma function:

$$
g(z)=\sum_{k=0}^{+\infty} \frac{w_{k}}{\Gamma(k+5 / 2)} z^{k+3 / 2}=z^{3 / 2} \sum_{k=0}^{+\infty} \frac{w_{k}}{\Gamma(k+5 / 2)} z^{k} .
$$

In general, this first series is ill-fitted for good numerical results because the terms $\frac{w_{k}}{\Gamma(k+5 / 2)} z^{k}$ have alternating signs and are much higher in absolute value than the actual value of $g(z)$. This problem of evaluating entire functions in finite precision arithmetic was previously addressed in $[7,6]$ and the same idea is used here. Function $g$ is multiplied by another function $h$ such that both $h$ and $h g$ have explicit power series with positive coefficients - which is good from a computational point of view. Since D-finite functions are closed by multiplication, choosing a D-finite $h$ results in a D-finite product $h g$, meaning that their power series coefficients satisfy an explicit linear recurrence. Let us do the following preconditioning:

$$
\exp \left(\frac{z}{\sigma_{3}^{2}}\right) g(z)=\sum_{k=0}^{+\infty} u_{k} z^{k}
$$

so that

$$
g(z)=\exp \left(-\frac{z}{\sigma_{3}^{2}}\right) \sum_{k=0}^{+\infty} u_{k} z^{k} .
$$

The linear recursive formula for $\left(u_{k}\right)_{k \geq 0}$ can be obtained in closed form using the recurrence defining $\left(w_{k}\right)_{k \geq 0}$. Is was done once again with Gfun. Getting back to the original probability, one immediately gets formula (23).

\section{Numerical results}

In this section, the proposed approach is applied on a test case presented by S. Alfano in [1, case 9] where he compares several methods to compute collision probabilities (but offers no avoidance strategy). This is not a real case scenario but it is supposed to be representative of two objects in highly eccentric Earth orbits (HEO).

The reference orbit of the primary object has a mean motion $n=1.4591 \times 10^{-4}$ $\mathrm{s}^{-1}$ and an eccentricity $e=0.741$. The origin of time $t=0$ is chosen as the nominal time of closest approach in the unmitigated case. This time corresponds to the instant when the mean relative distance reaches its minimal value if no maneuver is performed. This is typically around this time that the instantaneous collision proba- 
bility is the highest. The history of instantaneous collision probability, when no maneuver is performed, was computed using the method previously introduced. Figure 4 shows a close-up around its peak that looks similar to a plot provided by Alfano in [1], although a different model was used to propagate uncertainty in his paper. Clearly, the risk is very high as collision probability reaches near $t=0$ a maximum of 0.2813 (which is also very close to the value 0.2812 found in [1]).

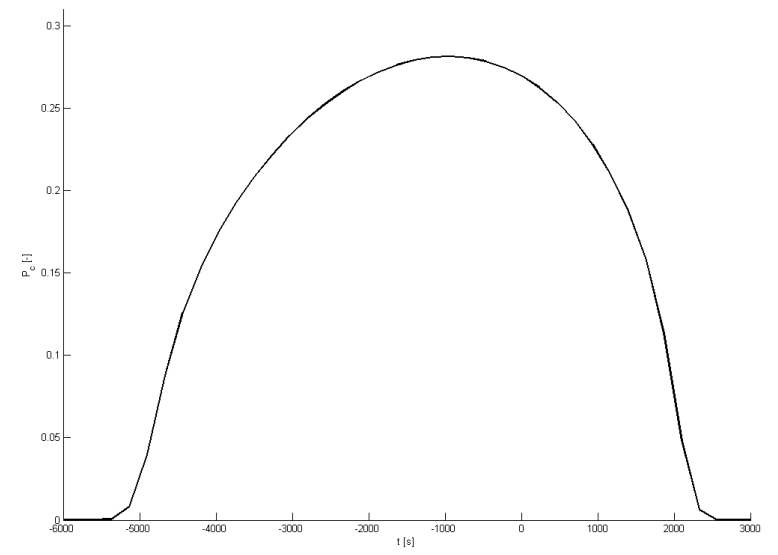

Fig. 4 Instantaneous collision probability as a function of time

For this reason, $t=0$ is chosen at the midpoint of the interval $\left[t_{0}, t_{f}\right]$, with $t_{0}=$ $-35,000 \mathrm{~s}$ and $t_{f}=+35,000 \mathrm{~s}$. The nominal angular position at initial time $t_{0}$ is $v_{0}=-3.071 \mathrm{rad}$. The mean primary position and velocity are assumed to be equal to the nominal one at $t_{0}$. Initial conditions for the relative state in the LVLH frame are given in Table 1 (mean vector) and Equation 32 (covariance matrix in meters and seconds). The combined radius of the objects is $R=6 \mathrm{~m}$ and the risk threshold is $\delta=$ $10^{-4}$. Both control grid $\left(N_{1}=50\right)$ and constrained grid $\left(N_{2}=200\right)$ are uniformly sampled.

\begin{tabular}{|c|c|}
\hline$\mu_{\mathbf{R}^{0}}(1)(\mathrm{m})$ & -46.830 \\
\hline$\mu_{\mathbf{R}^{0}}(2)(\mathrm{m})$ & 0 \\
\hline$\mu_{\mathbf{R}^{0}}(3)(\mathrm{m})$ & 2.986 \\
\hline$\mu_{\mathbf{V}^{0}}(1)\left(\mathrm{mm} \cdot \mathrm{s}^{-1}\right)$ & -0.643 \\
\hline$\mu_{\mathbf{V}^{0}}(2)\left(\mathrm{mm} \cdot \mathrm{s}^{-1}\right)$ & 0 \\
\hline$\mu_{\mathbf{V}^{0}}(3)\left(\mathrm{mm} \cdot \mathrm{s}^{-1}\right)$ & -1.922 \\
\hline
\end{tabular}

Table 1 Mean relative state at time $t_{0}$ 


$$
\left(\begin{array}{cccccc}
42.9181 & 0 & 2.5144 & 2.607 \times 10^{-3} & 0 & 8.0097 \times 10^{-3} \\
& 0.0649 & 0 & 0 & -0.0024 \times 10^{-3} & 0 \\
& & 0.1549 & 0.1574 \times 10^{-3} & 0 & 0.4794 \times 10^{-3} \\
& & & 0.1613 \times 10^{-6} & 0 & 0.4928 \times 10^{-6} \\
\text { sym } & & & 0.0001 \times 10^{-6} & 0 \\
& & & & & 1.5083 \times 10^{-6}
\end{array}\right)
$$

To limit the number of equations used to define the polyhedral approximation of the collision set and thus the computational time, the spherical combined object is over-approximated by the following cube:

$$
\left\{\left(r_{1}, r_{2}, r_{3}\right) \in \mathbb{R}^{3}:-r_{i} \leq R, r_{i} \leq R \quad \forall i=1,2,3\right\} .
$$

The overall implementation was done with Matlab (c) R2014a on an Intel $($ Xeon® at 3.60GHz. The mixed-integer linear program has been solved using Gurobi 5.6.0 [8] with a big-M of $10^{4} \mathrm{~m}$.

The solution is detailed in Table 2 and was obtained after $1.6 \mathrm{~s}$. It consists in only 4 non-negligible impulses. It is noticeable that - except for the first one - every maneuver has only one non-zero component and that there is no out-of-plane maneuvers. The corresponding fuel-cost is $J_{1}^{*}=4.4 \mathrm{~mm} / \mathrm{s}$.

\begin{tabular}{|c|c|c|c|c|}
\hline Number & Instant (s) & \multicolumn{4}{|c|}{ LLLH components (mm/s) } \\
\hline 1 & $-35,000$ & 0.0167 & 0 & -3.0360 \\
\hline 2 & 22,143 & 0.5167 & 0 & 0 \\
\hline 3 & 23,571 & 0.2777 & 0 & 0 \\
\hline 4 & 30,714 & -0.5493 & 0 & 0 \\
\hline
\end{tabular}

Table 2 Optimal impulses

Next Figures 5, 6, 7 and 8 illustrate the effects of the thrusting strategy. The primary mean position and velocity and the nonzero optimal impulses can be seen in Figures 5 and 6: as expected, the primary mean vector goes back to its nominal state at the end of the time horizon.

The history of instantaneous collision probability was also computed in the controlled case, on a tighter grid of 300 points (recall that it was 200 for the optimization algorithm) in $558.9 \mathrm{~s}$. It appears that the maximum risk is very close to the authorized $\delta$-threshold since the highest probability is $1.004 \times 10^{-4}$. Note that if the chance constraints are not satisfied on a thinner grid than the one used for the optimization, the algorithm could be run with a bigger value for $N_{2}$. Figure 7 depicts one hundred occurrences - randomly sampled - of the uncontrolled relative motion while Figure 8 shows the same trajectories when performing the maneuvers. One can see that the impulses allow to safely avoid the secondary object (in black) for all the trials whereas 33 of them lead to a collision when there is no control. 

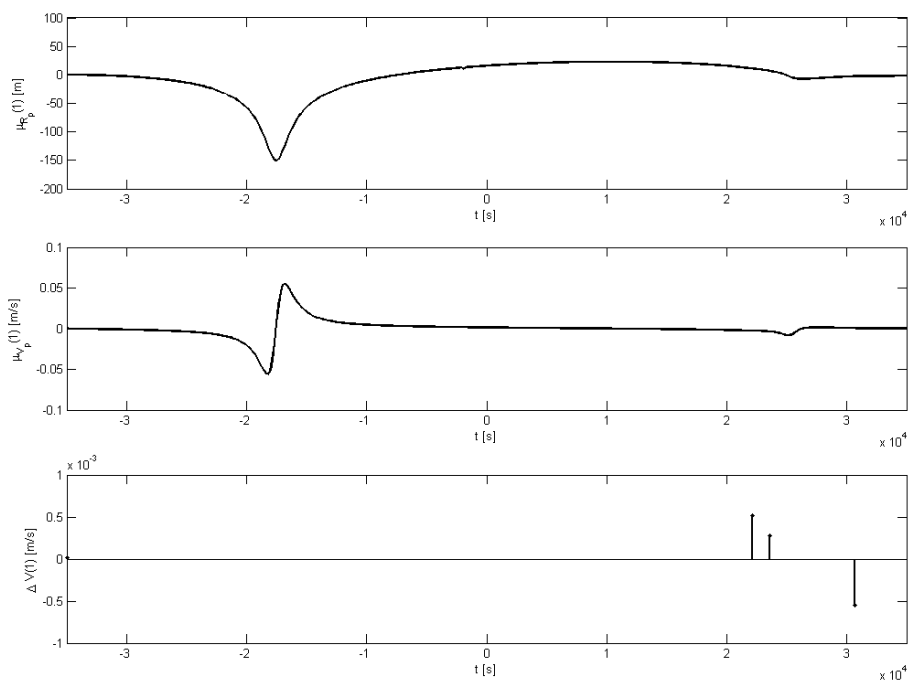

Fig. 5 Primary mean position, mean velocity and optimal impulses along the first LVLH axis versus time
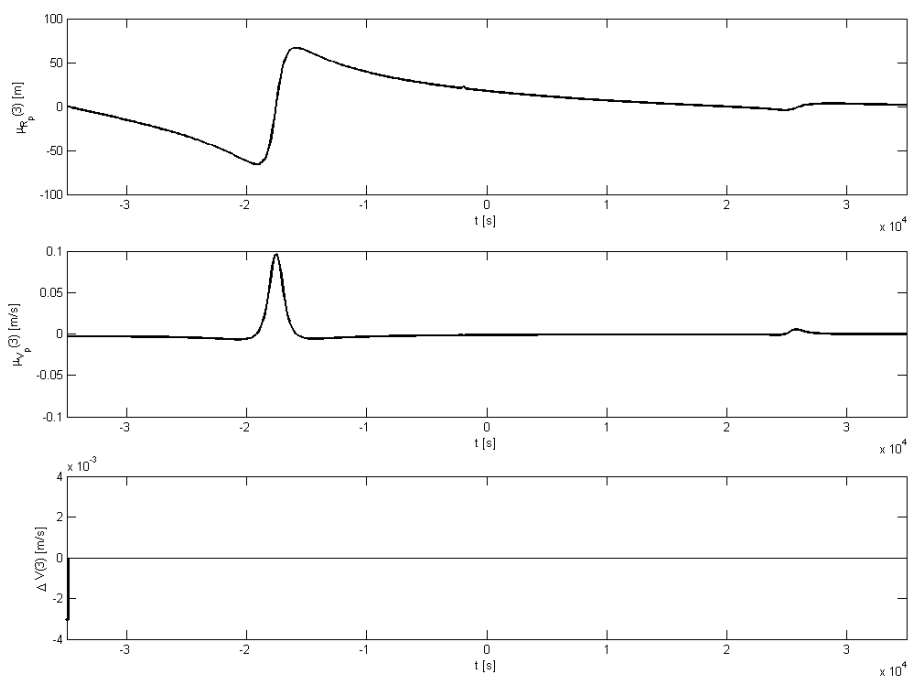

Fig. 6 Primary mean position, mean velocity and optimal impulses along the third LVLH axis versus time 


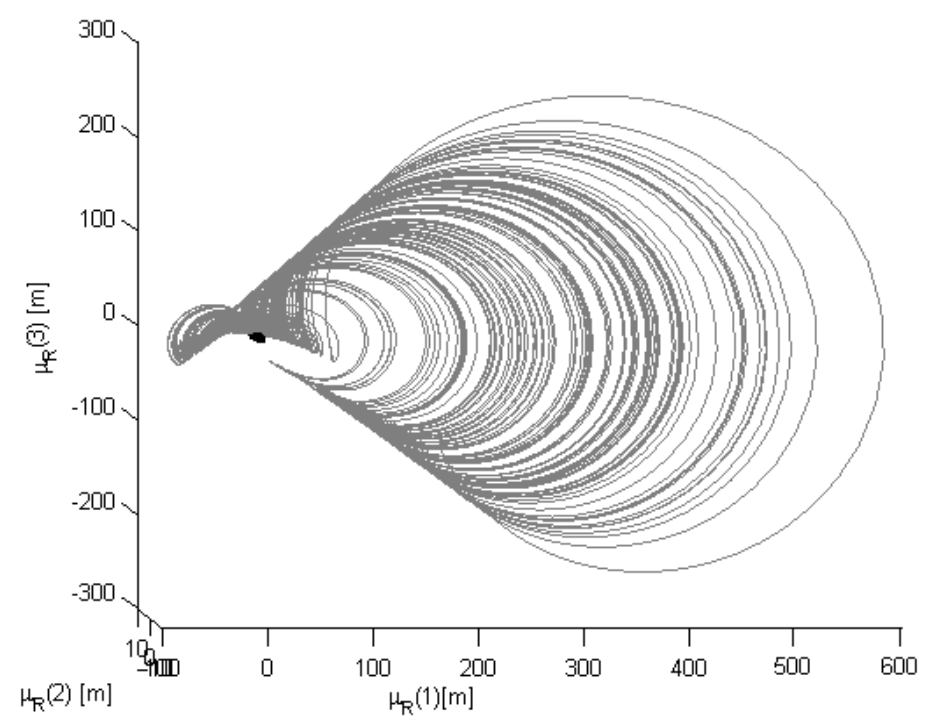

Fig. 7 Examples of uncontrolled relative trajectories of the primary over the encounter. The secondary object is in black.

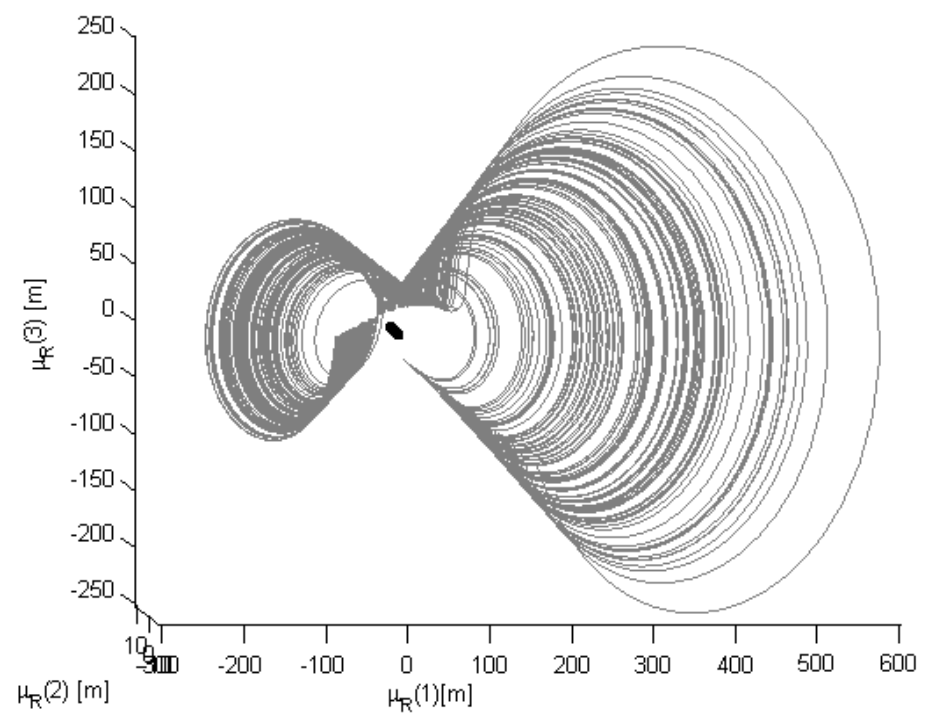

Fig. 8 Examples of controlled relative trajectories of the primary over the encounter. The secondary object is in black. 


\section{Conclusion}

This paper proposes a probabilistic formulation of the collision avoidance problem between an active spacecraft and an orbital debris as a joint chance constrained optimization problem. The framework of this study is restricted to long-term encounters, enabling the linearization of the equations of the relative dynamics. Since the original problem is not tractable, two relaxations are proposed: first the collision set is conservatively approximated by a convex polyhedron containing it, and then the joint chance constrained optimization problem is replaced by an individual chance constrained problem. The latter is equivalently reformulated as a deterministic disjunctive linear program which can be solved using a classical mixed-integer solver. To validate the proposed approach, a new method to efficiently compute 3-D Gaussian integrals on Euclidean balls is presented. It is then used to evaluate the instantaneous probability of collision for a spherical collision set at any time of the mission. The efficiency of the proposed approach is illustrated on a test case by computing the instantaneous probabilities of collision over the whole time horizon. It is numerically checked that the risk remains below the required tolerance threshold. The main limitation of the approach is that the probability of collision over the entire encounter is not directly dealt with in the proposed optimization process. So, there is no theoretical guarantee that the probability of collision remains below the required threshold all along the mission.

As far as the computation of the maneuvers is concerned, improvements can be obtained depending on mission requirements. For instance, an upper bound on the magnitude of the velocity increments can easily be added as linear constraints. The control law could also be restricted to in-plane maneuvers. Another perspective is to extend the collision avoidance problem to multiple active spacecraft (formation flying) and/or multiple debris (orbit safety). This could only be done for a reasonable number of additional objects as complexity would rise accordingly. Concerning the computation of the probability of collision, some improvements will be considered in short term. One perspective will be to find upper bounds on the truncation error and lower bounds on the probability when uncertainty varies. Such results should be of a great interest to retrieve valuable information from a mission point of view on collision probabilities for any type of space encounter.

\section{Acknowledgments}

The Authors would like to thank Alexandre Falcoz from Airbus Defence and Space for the grant that partly supports this activity. 


\section{References}

1. S. Alfano. A numerical implementation of spherical objet collision probability. Journal of Astronautical Sciences, 53(1), January-March 2005.

2. D. Bertsimas, J.N. Tsitsiklis. Introduction to linear optimization. AIAA. Athena Scientific, Belmont, Massachusetts, 1997.

3. L. Blackmore, H. Li, and B. Williams. A probabilistic approach to optimal robust path planning with obstacles. In American Control Conference, Minneapolis, MA, June 2006.

4. L. Blackmore, M. Ono, and B. Williams. Chance-constrained optimal path planning with obstacles. Robotics, IEEE Transactions on, 27(6):1080-1094, 2011.

5. F.K. Chan. Spacecraft Collision Probability. AIAA. The Aerospace Press, 2008.

6. S. Chevillard and M. Mezzarobba. Multiple-Precision Evaluation of the Airy Ai Function with Reduced Cancellation. In A. Nannarelli, P.-M. Seidel, and P. T. P. Tang, editors, 21st IEEE SYMPOSIUM on Computer Arithmetic, pages 175-182, Los Alamitos, CA, Avril 2013. IEEE Computer Society.

7. W. Gawronski, J. Müller, and M. Reinhard. Reduced cancellation in the evaluation of entire functions and applications to the error function. SIAM Journal on Numerical Analysis, 45(6):2564-2576, 2007.

8. Gurobi Optimization, Inc., Gurobi Optimizer Reference Manual, http://www.gurobi.com, 2014.

9. J.B. Lasserre and E.S. Zeron. Solving a class of multivariate integration problems via laplace techniques. Applicationes Mathematicae, 2001.

10. J.B. Mueller and R. Larsson. Collision avoidance maneuver planning with robust optimization. In 7th International ESA Conference on Guidance, Navigation and Control Systems, 2008.

11. R.P. Patera and G.E. Peterson. Space vehicle maneuver method to lower collision risk to an acceptable level. Journal of Guidance, Control and Dynamics, 26(2), March-April 2003.

12. A. R. Richards, T. Schouwenaars, J. P. How, and E. Feron. Spacecraft trajectory planning with avoidance constraints using mixed-integer linear programming. Journal of Guidance, Control and Dynamics, 25(4), August 2002.

13. B. Salvy. D-finiteness: Algorithms and applications. In Manuel Kauers, editor, ISSAC 2005: Proceedings of the 18th International Symposium on Symbolic and Algebraic Computation, Beijing, China, July 24-27, 2005, pages 2-3. ACM Press, 2005. Abstract for an invited talk.

14. B. Salvy and P. Zimmermann. Gfun: a Maple package for the manipulation of generating and holonomic functions in one variable. ACM Transactions on Mathematical Software, 20(2):163-177, 1994.

15. N. Sánchez-Ortiz, M. Belló-Mora, and H. Klinkrad. Collision avoidance manoeuvres during spacecraft mission lifetime: Risk reduction and required $\delta v$. Advances in Space Research, 38(9):2107-2116, 2006.

16. R. Serra, D. Arzelier, J-B. Lasserre, M. Joldes, and A. Rondepierre. A new method to compute the probability of collision for short-term space encounters. In Proceedings of AAS/AIAA Astrodynamics Specialist Conference, San Diego, California, USA, August 2014.

17. G.L. Slater, S.M. Byram, and T.W. Williams. Collision avoidance for satellites in formation flight. Journal of guidance, control, and dynamics, 29(5):1140-1146, 2006.

18. J. Tschauner and P. Hempel. Optimale beschleunigungs-programme fur des rendezvous manover. Astronautica Acta, 5-6:296-307, 1964.

19. D.V. Widder. An introduction to transform theory. Academic Press New York, 1971.

20. K. Yamanaka and F. Ankersen. New state transition matrix for relative motion on an arbitrary elliptical orbit. Journal of Guidance, Control and Dynamics, 25(1), january 2002.

21. D. Zeilberger. A holonomic systems approach to special functions identities. Journal of Computational and Applied Mathematics, 32(3):321-368, 1990. 